\title{
ATIVIDADE ELETROMIOGRÁFICA DE ESTABILIZADORES DE TRONCO NO EXERCÍCIO PUSH-UP ISOMÉTRICO COM VIBRAÇÕES MECÂNICAS
}

\author{
ELECTROMIOGRAPHIC ACTIVITY OF TRUNK STABILIZERS IN THE \\ ISOMETRIC PUSH-UP EXERCISE WITH MECHANICAL VIBRATIONS
}

\section{ACTIVIDAD ELECTROMIOGRÁFICA DE ESTABILIZADORES DE TRONCO EM EL EJERCICIO DE ELEVÁCION ISOMÉTRICA COM VIVRACIONES MECÁNICAS}

\author{
Victor Costa Coutinho \\ https://orcid.org/0000-0003-0773-5029 (i) \\ http://lattes.cnpq.br/2564137376074193 \\ Universidade Federal de Mato Grosso (Cuiabá, MT - Brasil) \\ victorcosta.vtf@gmail.com \\ Patrick dos Santos \\ https://orcid.org/0000-0002-3271-4564 id \\ http://lattes.cnpq.br/1615572275801178 \\ Universidade Federal de Mato Grosso (Cuiabá, MT - Brasil) \\ patrick28547@gmail.com \\ Fabio Ferreira da Silva \\ https://orcid.org/0000-0002-9293-7970 \\ http://lattes.cnpq.br/0194126355970916 \\ Universidade Federal de Mato Grosso (Cuiabá, MT - Brasil) \\ fabio_1-7@hotmail.com \\ Jacielle Carolina Ferreira \\ https://orcid.org/0000-0002-9321-749X (D) \\ http://lattes.cnpq.br/4097604888377977 \\ Universidade Federal de Mato Grosso (Cuiabá, MT - Brasil) \\ jacielleferreira@gmail.com
}

\section{Resumo}

O objetivo desse estudo foi verificar se a vibração mecânica aumenta a ativação eletromiográfica (EMG) de músculos do core durante o exercício de push-up isométrico. Participaram do estudo 13 homens saudáveis. As sessões experimentais consistiram na realização de uma repetição de push-up isométrico com angulação dos cotovelos a $90^{\circ}$ até a exaustão voluntária. Em uma sessão, o push-up era realizado com as mãos sobre uma plataforma vibratória a uma frequência de $20 \mathrm{~Hz}\left(\mathrm{~V}_{20}\right)$. Na outra sessão a plataforma permanecia desligada durante a realização do exercício $\left(V_{00}\right)$. Avaliou-se a EMG dos músculos Reto Abdominal (RA) e Longuíssimo (LO). O Root Mean Square (RMS) do RA e do LO foi maior na $\mathrm{V}_{20}$ comparada à $\mathrm{V}_{00}$ (RA: $p=0,002 ; g=0,6 ; \mathrm{LO}: \mathrm{p}=0,001 ; g=1,2$ ), assim como a Frequência Mediana ( $F_{M E D)}(R A: p=0,004 ; g=2 ; L O: p=0,001 ; g=2$ ). A vibração mecânica alterou as respostas neuromusculares do core, visto que a EMG dos músculos analisados foi maior na sessão $V_{20}$ comparada à sessão $\mathrm{V}_{00}$.

Palavras-chave: Core; Reto Abdominal; Longuíssimo.

\section{Abstract}

The aim of this study was to verify whether mechanical vibration increases the electromyographic activation (EMG) of the core muscles during the isometric push-up exercise. Thirteen healthy men participated in the study. The 
experimental sessions consisted of performing a repetition of isometric push-up with elbow angles at $90^{\circ}$ until voluntary exhaustion. In one session, the push-up was performed with the hands on a vibrating platform at a frequency of $20 \mathrm{~Hz}\left(\mathrm{~V}_{20}\right)$. In the other session, the platform remained off during the exercise $\left(\mathrm{V}_{00}\right)$. The EMG of the Rectus Abdominal (RA) and Longissimus (LO) muscles was evaluated. The Root Mean Square (RMS) of RA and LO was higher at $\mathrm{V}_{20}$ compared to $\mathrm{V}_{00}(\mathrm{RA}: \mathrm{p}=0.002 ; g=0,6 ; \mathrm{LO}: \mathrm{p}=0.001 ; g=1,2$ ), as well as Median Frequency (FMED) (RA: $p=0.004 ; g=2 ; \mathrm{LO}: \mathrm{p}=0.001 ; g=2)$. The mechanical vibration altered the neuromuscular responses of the core, since the EMG of the muscles analyzed was greater in the $\mathrm{V}_{20}$ session compared to the $\mathrm{V}_{00}$ session.

Keywords: Core; Rectus Abdominal; Longissimus.

\section{Resumen}

El objetivo de este estudio fue verificar si la vibración mecánica aumenta la activación electromiográfica (EMG) de los músculos de core durante el ejercicio de flexión isométrica. Trece hombres participaron en el estudio. Las sesiones experimentales consistieron en realizar una repetición de flexiones isométricas con ángulos de codo a $90^{\circ}$ hasta el agotamiento voluntario. En una sesión, el push-up se realizó con las manos sobre una plataforma vibratoria a una frecuencia de $20 \mathrm{~Hz}\left(\mathrm{~V}_{20}\right)$. En la otra sesión, la plataforma permaneció apagada durante el ejercicio $\left(\mathrm{V}_{00}\right)$. Se evaluó la EMG de los músculos recto abdominal (RA) y Longissimus (LO). La raíz cuadrada media (RMS) de RA y LO fue mayor en V20 en comparación con V00 (RA: $p=0,002 ; g=0,6 ; \mathrm{LO}: p=0,001 ; g=1,2$ ), así como la frecuencia media (FMED) (RA: $p=0,004 ; g=2 ; L O: p=0,001 ; g=2$ ). La vibración mecánica alteró las respuestas neuromusculares del core, ya que la EMG de los músculos analizados fue mayor en la sesión V20 en comparación con la sesión $V_{00}$.

Palabras clave: Core; Rectus Abdominal; Longuíssimo.

\section{INTRODUÇÃO}

Os músculos estabilizadores do tronco compõem o grupo muscular denominado core, dentre esses músculos, estão Longuíssimo (LO) e Reto abdominal (RA), que são utilizados na realização de atividades diárias e esportivas que requerem uma estabilidade dinâmica ou estática do tronco. Os estabilizadores são importantes devido à sua capacidade de absorver, produzir e transportar forças multidirecionais mantendo a estabilidade e mobilidade. A existência de déficits nas capacidades de força e controle motor tendem a diminuir a estabilização, aumentando o risco de lesão (PALMER et al., 2001).

Os meios de treinamento dos músculos estabilizadores têm como objetivo potencializar a mobilidade distal, através de uma melhor estabilização proximal. Eles geralmente envolvem isometria de baixa intensidade e sincronia dos músculos profundos do tronco (BARR et al., 2005; TEYHEN et al., 2008).

Reinher e colaboradores (2008) propõem que o treino de estabilização seja subdividido em quatro estágios sendo o primeiro voltado ao recrutamento dos músculos abdominais, além do aprendizado da manutenção da pelve em posição neutra. No segundo estágio ocorre a correção dos desequilíbrios de força e resistência muscular, enquanto no terceiro estágio viabiliza e requisita a reeducação dinâmica dos músculos estabilizadores e no quarto e último estágio são executados exercícios avançados de estabilização dinâmica. 
A demanda por estabilidade pode ser promovida, dentre outros meios, pela vibração mecânica, como aplicada no estudo de Youdas e colaboradores (2017) que avaliaram as ativações musculares do abdômen, musculaturas da parte posterior e lateral do tronco durante o exercício de prancha em diferentes posições e uma bola com vibração para gerar o desequilíbrio. De acordo com Rodrigues e colaboradores (2016) a vibração obtida através da utilização da plataforma vibratória tem a capacidade de promover a desestabilização do centro de gravidade, exercitando assim o equilíbrio estático do indivíduo por meio da estimulação dos receptores sensoriais, com o propósito de ativar a resposta muscular reflexa para neutralizar as vibrações. Assim, além do efeito neuromuscular esperado na musculatura alvo que recebe o input vibratório, a vibração mecânica ainda pode ser explorada como meio de promoção de instabilidade para músculos que não recebem diretamente a vibração mecânica proveniente dos diferentes equipamentos capazes de gerar esse tipo de estímulo.

A vibração é um estímulo mecânico caracterizado por movimentos oscilatórios, sendo mensurada em Hertz $(\mathrm{Hz})$, que representa o número de ciclos de movimento por segundo. O uso da vibração mecânica tem se destacado como um método auxiliar no treinamento de capacidades físicas para aumento do ganho de força, flexibilidade, resistência e potência com a utilização de equipamentos vibratórios (HALLAL et al., 2010). Usualmente, a fonte de vibração nos estudos na área da atividade física é a plataforma vibratória, com frequências que variam de 10 até $60 \mathrm{~Hz}$. A plataforma vibratória promove a vibração de corpo inteiro $(\mathrm{VCl})$ e devido à sua versatilidade, proporciona a realização de diversos protocolos de treinamento com inúmeras combinações de frequência, amplitude e tempo de exposição (HALLAL et al., 2010).

Os primeiros relatos advindos dos estudos com vibração mostraram que a vibração promove contrações involuntárias reflexas, especialmente o reflexo tônico de vibração (EKLUND e HAGBARTH, 1966). Tal reflexo ocorre através do aumento da taxa de disparo das fibras aferentes do tipo la, causado pela expansão no comprimento do fuso muscular, gerando como resposta final um maior número de excitações dos neurônios motores alpha e consequentemente a contração de unidades motoras (SHINOHARA, 2005). A partir da observação dos efeitos da vibração no sistema neuromuscular, o uso de plataformas vibratórias foi ampliado e o efeito da vibração no sistema neuromuscular passou a ser analisado principalmente nos músculos dos membros inferiores em exercícios de agachamento, como nos estudos de Cardinale e Lim (2003); Cochrane (2011), que utilizaram a VCl, enquanto poucos 
estudos avaliaram os efeitos da vibração em músculos do tronco, ou dos membros superiores (ASHNAGAR et al., 2016; COCHRANE et al., 2014). Assim, apesar da possibilidade de a vibração atuar como estímulo promotor de desestabilização, pouco se sabe sobre os efeitos neuromusculares da sua aplicação nos músculos estabilizadores do tronco.

Diante da possibilidade de as plataformas vibratórias apresentarem-se como recurso adicional para promoção de desestabilidade durante o treinamento dos músculos estabilizadores do tronco e da necessidade de uma maior compreensão sobre a ativação dos músculos estabilizadores do tronco sob estímulo vibratório, o presente estudo teve como objetivo verificar se a vibração mecânica aumenta a ativação eletromiográfica dos músculos estabilizadores do tronco durante o exercício de push-up isométrico.

\section{METODOLOGIA}

\section{Amostra e cuidados éticos}

Participaram deste estudo 13 homens praticantes de exercício resistido há mais de quatro meses, com média de idade de 25,52 $\pm 4,44$ anos, estatura de $175 \pm 4,74 \mathrm{~cm}$, massa de $78,48 \pm 12,81 \mathrm{~kg}$ e Índice de Massa Corporal (IMC) de 23,75 $\pm 3,81 \mathrm{~kg} / \mathrm{m}^{2}$. Os indivíduos selecionados não possuíam qualquer tipo de lesão, histórico de cirurgia ou prótese no aparelho locomotor, assim como não eram hipertensos ou portadores de marca-passo. Todos assinaram o Termo de Consentimento Livre e Esclarecido e a pesquisa seguiu as recomendações éticas locais e está de acordo com a Declaração de Helsinki (1995). O estudo foi aprovado pelo Comitê de Ética em Pesquisa da Universidade Federal de Mato Grosso sob $\mathrm{n}^{\circ}$ 33016620.3.0000.8124.

\section{Procedimentos e Materiais}

Os voluntários compareceram ao laboratório três vezes, com um intervalo mínimo de 48 horas entre cada visita. Na primeira, houve a demarcação dos pontos em que seriam posicionados os eletrodos de cloreto de prata (MSGST-06, Solidor ${ }^{\circledR}$ ) nos músculos RA e LO, seguindo o estudo de NG e colaboradores (2002).

Após demarcação do local dos eletrodos, os voluntários foram informados sobre o protocolo e o posicionamento a ser adotado no exercício de push-up isométrico (figura 1), 
que consistiu em manter o corpo estendido em decúbito ventral, mãos paralelas na linha horizontal do processo xifoide e cotovelos em $90^{\circ}$, sendo este ângulo encontrado através de um goniômetro manual. Para auxiliar no posicionamento, foi utilizado um step da mesma altura da plataforma vibratória a fim de manter o alinhamento horizontal do corpo do voluntário (figura 1). Passadas as informações acerca da padronização do exercício, os voluntários realizaram um aquecimento específico, com duas séries de 12 a 15 push-ups dinâmicos e então foram familiarizados com o exercício sobre a plataforma vibratória (P204, KIKOS). No processo de familiarização com o exercício, cada voluntário executou duas séries de 15 segundos sem vibração (plataforma vibratória desligada), e finalizou com duas séries de quinze segundos com vibração de $20 \mathrm{~Hz}$ (mesma frequência utilizada na sessão experimental). O intervalo entre séries foi de 45 segundos. Ao final da primeira sessão, os voluntários foram instruídos a não lavar a marcação realizada nos músculos avaliados e não praticar exercícios físicos nas 24 horas antecedentes às próximas sessões experimentais

Figura 1 - Posicionamento do voluntário para o exercício push-up

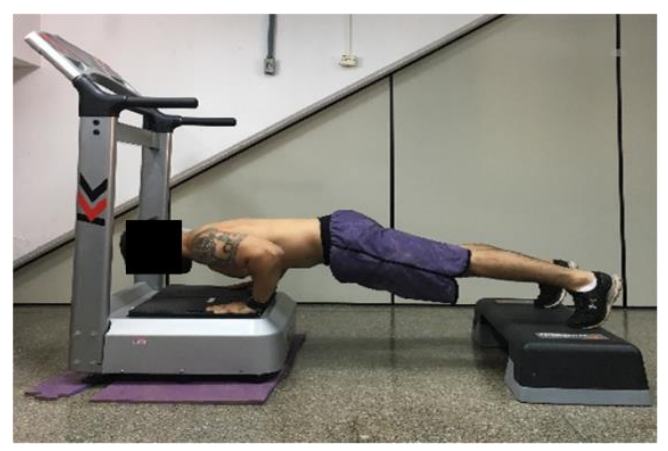

Fonte: acervo dos autores.

As sessões seguintes consistiram na realização do protocolo experimental, sendo uma sem vibração $\left(V_{00}\right)$ e outra com vibração mecânica de $20 \mathrm{~Hz}\left(\mathrm{~V}_{20}\right)$. O primeiro voluntário iniciou com a sessão $V_{00}$, o segundo com a sessão $V_{20}$, o terceiro com a sessão $V_{00} e$, assim sucessivamente. As sessões experimentais consistiram em aquecimento geral e específico similares aos executados no dia da familiarização, seguido da tricotomia e assepsia do local demarcado no RA e LO, para então fixar os eletrodos e dar início ao teste. O teste consistiu na realização de uma repetição do exercício extensão isométrica de cotovelos a 90, em que o voluntário deveria permanecer até a exaustão voluntária, ou até o momento em que o indivíduo não conseguisse manter os cotovelos a $90^{\circ}$ (analisado por goniômetro manual). 
Durante o teste o tempo foi marcado com cronômetro digital e os sinais eletromiográficos do RA e LO foram captados e gravados por um eletromiógrafo de oito canais (EMG 830C, EMG System $\left.{ }^{\circledR}\right)$.

Ao final dos procedimentos da segunda visita, o local de posicionamento dos eletrodos foi reforçado com caneta dermográfica e os voluntários foram instruídos a reforçarem o local demarcado até a terceira e última sessão da coleta de dados. O intervalo entre as sessões de coleta de dados foi de 48 a 96 horas.

\section{Redução dos dados}

A aquisição dos dados da eletromiografia foi realizada a uma frequência de $2000 \mathrm{~Hz}$ e amplificados com ganho de 500. Nos sinais, foram aplicados filtros Butterworth de segunda ordem passa-faixa entre 10 e $500 \mathrm{~Hz}$, e rejeita-faixa de $20 \mathrm{~Hz}$ e $60 \mathrm{~Hz}$. Em seguida foi calculada a Root Mean Square (RMS) e a frequência mediana ( $\left.F_{M E D}\right)$ dos sinais eletromiográficos do RA e do LO, utilizando o sinal completo e o sinal parcial em dois momentos temporais, que foram os 10 segundos iniciais (10i) e finais (10f) do exercício, uma vez que o exercício foi até a falha, cada voluntário apresentou um tempo total de exercício diferente. Para cada voluntário, a duração do sinal completo tem um valor diferente pelo fato de os mesmos não executarem o exercício pelo mesmo tempo.

\section{Análise Estatística}

Os dados estão descritos como média e desvio padrão. A normalidade da distribuição dos dados foi confirmada pelo teste de Shapiro-Wilk. Para comparação dos valores de RMS e $F_{M E D}$ do sinal completo entre as sessões $V_{00}$ e $V_{20}$, utilizou-se o teste t-pareado. Para análise parcial do sinal RMS e $F_{M E D}$ entre os 10 segundos iniciais e finais do exercício e entre as sessões $V_{00}$ e $V_{20}$ utilizou-se ANOVA two-way (2x2) de medidas repetidas com post-hoc de Holm-Sidak, o tamanho do efeito foi calculado por meio do g de Edges $(g)$. O nível de significância adotado foi de $p<0,05$ e o pacote estatístico utilizado foi o Sigma Stat v. 3.5.

\section{RESULTADOS}

Na análise do sinal completo, a RMS do RA e do LO apresentaram-se maiores na sessão $\mathrm{V}_{20}$ comparada à $\mathrm{V}_{00}(\mathrm{p}=0,002 ; g=0,6$ e $p=0,001 ; g=1,2$, respectivamente), assim como 
a F FED de ambos os músculos (RA: $p=0,004 ; g=2 ; L O: p=0,001 ; g=2$ ). Desse modo, verifica-se que a vibração mecânica alterou as respostas neuromusculares, visto que a atividade elétrica EMG dos músculos foi maior na sessão $V_{20}$ comparada à sessão $V_{00}$. Os dados estão apresentados na tabela 1 .

Tabela 1 - Dados eletromiográficos referentes ao sinal completo

\begin{tabular}{lcccc}
\hline & \multicolumn{2}{c}{ RMS (mv) } & \multicolumn{2}{c}{ F $_{\text {MED }}$ (Hz) } \\
& V00 & V20 & Vo0 & V20 \\
\hline RA & $20,09 \pm 14,79$ & $32,10 \pm 22,57^{*}$ & $82,97 \pm 19,38$ & $111,14 \pm 26,72^{*}$ \\
LO & $16,01 \pm 6,23$ & $31,55 \pm 9,30^{*}$ & $89,74 \pm 10,72$ & $113,16 \pm 12,53^{*}$ \\
\hline
\end{tabular}

Nota: $\mathrm{RMS}=$ Root Mean Square; $\mathrm{F}_{\mathrm{MED}}=$ Mediana da Frequência; $\mathrm{V}_{00}=$ Sessão sem vibração; $\mathrm{V}_{20}=$ Sessão com vibração; RA = Reto Abdominal; $\mathrm{LO}=$ Longuíssimo; * Diferença em relação a $\mathrm{V}_{00}(\mathrm{p}<0,05)$.

Fonte: construção dos autores.

Na análise do sinal parcial, a ANOVA apontou que a RMS e $F_{M E D}$ não apresentaram interação entre as variáveis independentes (vibração e tempo) para ambos os músculos. Houve efeito principal de vibração para a RMS, sendo que a atividade eletromiográfica do RA e LO foi maior na sessão $V_{20}$, comparada à $V_{00}(R A: p=0,015$; $L O: p=0,002)$, assim como para a $F_{M E D}$ de ambos os músculos, que foi maior na sessão $V_{20}$ comparada a $V_{00}$ (RA: $p=0,002 ;$ LO: $p<0,001$ ). Também houve efeito principal de tempo para a RMS de ambos os músculos, visto que a RMS foi maior nos $10 f$ comparado aos 10i do exercício (RA: $p=0,025$; LO: $p=0,004)$. Entretanto, com relação à $\mathrm{F}_{\mathrm{MED}}$, houve efeito principal de tempo apenas para o músculo LO, com valor significativamente menor nos $10 \mathrm{f}$ comparado aos 10i do exercício independente da situação experimental $(p=0,019)$. Não houve efeito principal de tempo para a $F_{M E D}$ do músculo RA $(p=$ 0,11). Os resultados de RMS e $F_{\text {MED }}$ são apresentados nas figuras 2 e 3 . 
Figura 2 - Dados da RMS referentes à análise do sinal parcial do músculo Longuíssimo (a) e Reto abdominal (b) nas situações com vibração $\left(V_{20}\right)$ e sem vibração $\left(V_{00}\right)$, nos dez segundos inicias (10i) e nos dez segundos finais(10f) do exercício. Nota: *Indica diferença significativa em relação a $V_{00}$, Indica diferença significativa em relação aos $10 \mathrm{i}$.

a)

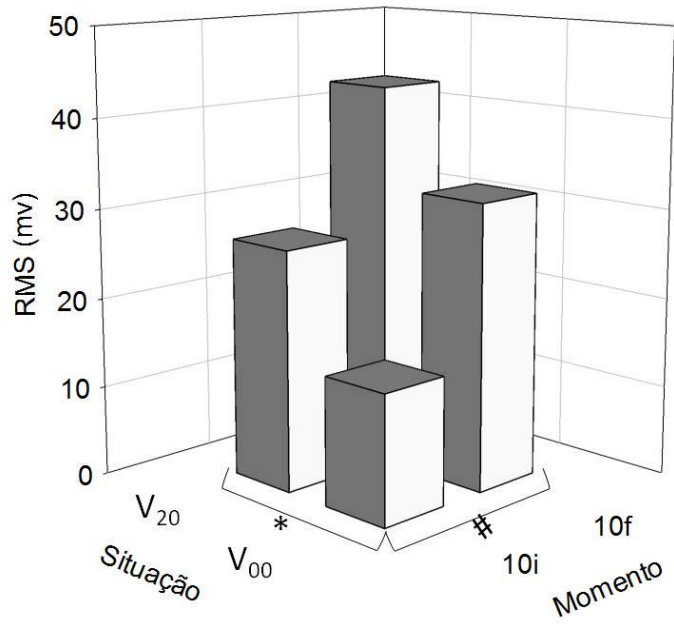

b)

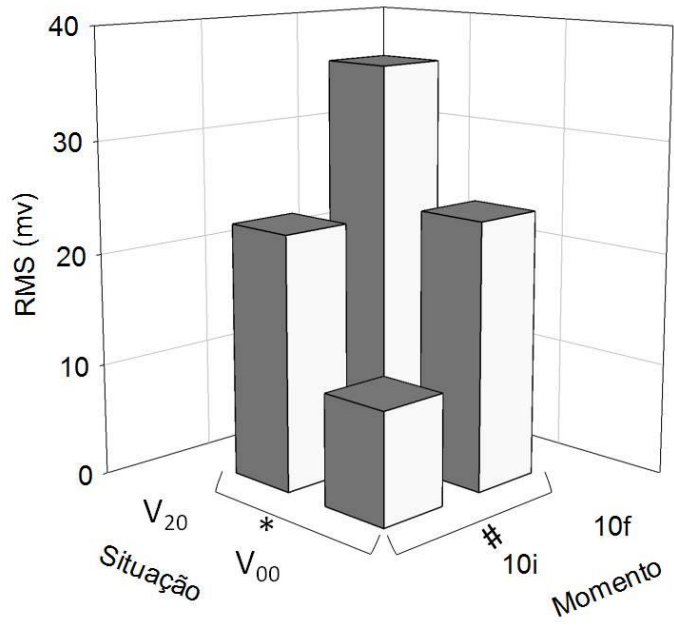

Fonte: construção dos autores.

Figura 3 - Dados da Frequência mediana $\left(F_{\mathrm{MED}}\right)$ referentes à análise do sinal parcial do músculo Longuíssimo (a) e Reto abdominal (b) nas situações com vibração $\left(V_{20}\right)$ e sem vibração $\left(V_{00}\right)$, nos dez segundos iniciais (10i) e nos dez segundos finais (10f) do exercício. * Indica diferença significativa em relação a $\bigvee_{00}$; \# Indica diferença significativa em relação aos $10 i$.
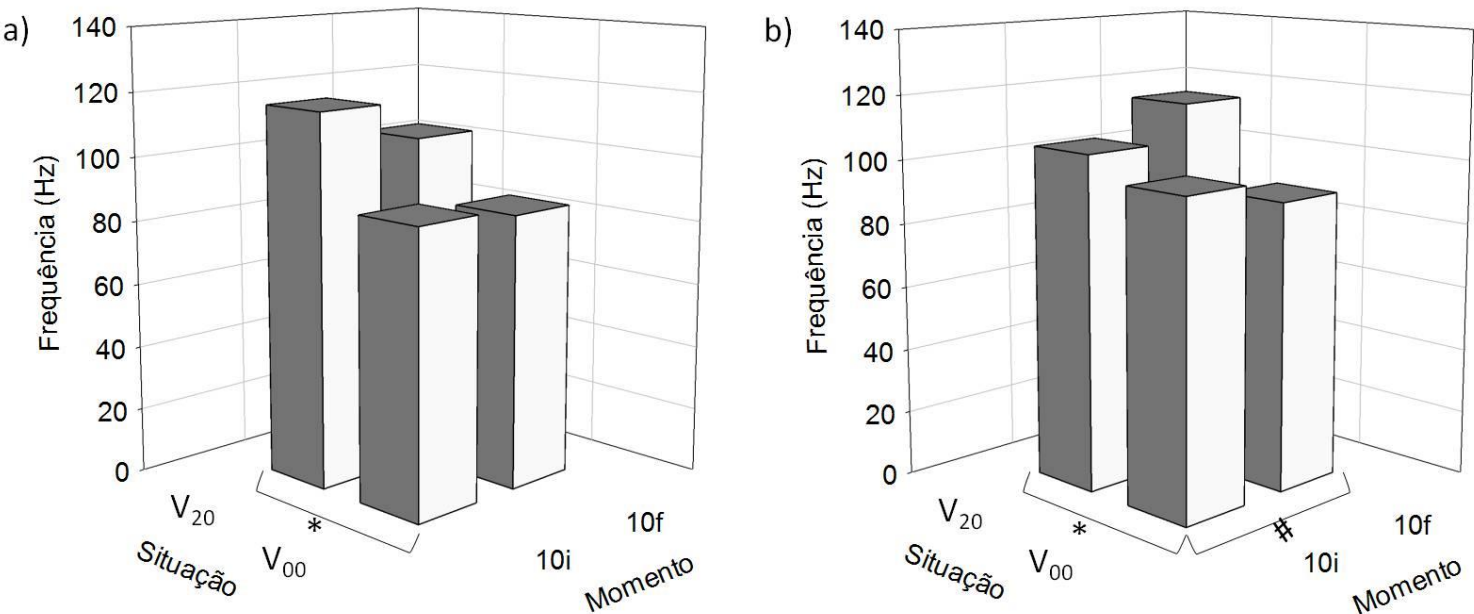

Fonte: construção dos autores. 


\section{DISCUSSÃO}

O objetivo do presente estudo foi verificar se a vibração mecânica aumenta ativação eletromiográfica dos músculos estabilizadores do tronco durante o exercício de pushup isométrico. Os resultados mostram que a adição da vibração durante o exercício foi capaz de aumentar a atividade neuromuscular de ambos os músculos analisados, independente do tempo de exercício analisado. Os resultados do presente estudo corroboram para a hipótese de que as vibrações mecânicas promovem maior demanda de atividade neural nos músculos do core, representado pela maior atividade eletromiográfica observada. Os músculos do core tem papel fundamental na manutenção do alinhamento articular da coluna vertebral, fornecendo suporte em situações estáticas e dinâmicas (PALMER et al., 2001). O uso da plataforma vibratória no treinamento do core aparece com o objetivo de intensificar a demanda neural, aumentando o recrutamento das unidades motoras e colaborando com o aumento da ativação neuromuscular (RODRIGUES et al., 2016).

Para estudar se a vibração tem impacto na atividade muscular, vários estudos mensuraram a EMG durante o treinamento com vibração. No entanto, a maioria dos estudos com EMG enfatizaram os músculos dos membros inferiores (CARDINALE; LIM, 2003; VERSCHUEREN, 2004; ROELANTS et al., 2006) e poucos estudos investigaram a atividade muscular dos músculos do core durante o treinamento com vibração.

Com relação aos músculos do core avaliados por meio da EMG, existem poucos estudos na literatura que investigaram o impacto da vibração sobre eles. Wirth e colaboradores (2011) avaliaram os efeitos da VCl sobre os músculos do core em 25 sujeitos de ambos os sexos, utilizando uma frequência de $30 \mathrm{~Hz}$ e 8 exercícios estáticos. Assim como em nosso estudo, os pesquisadores relataram que houve um aumento nos valores de RMS, mas em contraste com o presente estudo que utilizou somente um exercício estático, foram realizados 3 diferentes exercícios para o core na plataforma, sendo que os efeitos mais altos foram encontrados nos exercícios em que os sujeitos estavam diretamente sentados na plataforma. Isso pode ser explicado pela influência que a distância entre os músculos alvos e a superfície de contato com a plataforma exerce sobre a resposta neuromuscular (ROELANTS et al., 2006). Em outro estudo do mesmo autor foi investigado os efeitos agudos da $\mathrm{VCl}$ nos músculos posteriores do tronco e na atividade muscular abdominal em uma amostra similar ao do presente estudo. A adição de vibração resultou em aumentos significativos na atividade 
muscular, principalmente nos exercícios para a musculatura abdominal corroborando com os achados do presente estudo.

O impacto crônico do treinamento dos músculos do core utilizando vibrações mecânicas ainda não está bem estabelecido. Em estudos acerca da funcionalidade do core, observa-se diferentes resultados como no estudo de Osawa e colaboradores (2011) que avaliaram a força e resistência dos músculos anteriores e posteriores do core com treinamento de vibração em adultos saudáveis e concluíram que o exercício com adição da vibração, quando comparado ao exercício sem vibração, não promove mudanças na força e resistência muscular. Por outro lado, Maeda e colaboradores (2016) analisaram oito semanas de treinamento com vibração sobre o desempenho da força extensora e flexora de tronco e sua influência em testes funcionais. Os resultados mostraram diferença significativa na força dos músculos flexores do tronco quando os indivíduos eram expostos à vibração em comparação a situação sem vibração.

Apesar da maior atividade eletromiográfica observada no presente estudo, corroborando outros estudos agudos que encontraram resultados similares, os possíveis benefícios crônicos dessa maior demanda neural agudam durante exercícios com vibrações mecânicas ainda precisa ser investigado. Além disso, outros parâmetros biomecânicos como deslocamentos pico a pico e diferentes posições/ângulos articular e adotados no protocolo podem alterar o impacto da vibração mecânica sobre a ativação muscular. O estudo de Perchthaler e colaboradores (2015) por exemplo, verificou que a atividade muscular do tronco e pescoço durante $\mathrm{VCl}$ foi significativamente maior em relação à condição sem vibração, sendo que os maiores valores de ativação foram encontram em amplitudes pico a pico mais elevada, assim como houve influência da condição ângulo do joelho para ativação dos músculos das costas. Sendo assim, deve-se ter cautela com relação aos resultados do nosso estudo, sabendo que tais parâmetros biomecânicos assim como o protocolo adotado podem interferir no resultado da ativação muscular do core.

\section{CONCLUSÃO}

Do ponto de vista da prática, sugere-se que a vibração mecânica possa ser utilizada como meio de promover maior ativação dos músculos estabilizadores do tronco no exercício push-up isométrico. A variação para outros tipos de exercício e ações musculares ainda precisa 
ser investigada. Adicionalmente, o treinador deve levar em consideração os parâmetros mecânicos, como a frequência e o tipo de vibração aplicada, uma vez que, a alteração desses parâmetros pode alterar a resposta do sistema neuromuscular.

\section{REFERÊNCIAS BIBLIOGRÁFICAS}

ASHNAGAR, Zinat e colaboradores. The effects of whole-body vibration on EMG activity of the upper extremity muscles in static modified push up position. Journal of back and Musculoskeletal rehabilitation, v. 29, p. 557-563, 2016.

BARR, Karen; GRIGGS, Miriam; CADBY, Todd. Lumbar Stabilization. American journal physical medicine and rehabilitation, v. 84, n. 6, p. 473-480, 2005.

CARDINALE, Marco; LIM, Jon. Electromyography activity of vastus lateralis muscle during whole-body vibrations of different frequencies. Journal of strength conditioning research, v. 17, n. 3, p. 621-624, 2003.

COCHRANE, Darryl. The potential neural mechanisms of acute indirect vibration. Journal of sports science e medicine, v. 10, p. 19-30, 2011.

COCHRANE, Darryl. BLACK, Melissa; BARNES, Matthew. Does acute side-alternating vibration exercise enhance ballistic upper-body power? International journal of sports science and medicine, v. 35, n. 12, p. 1006-1011, 2014.

EKLUND, Greta; HAGBARTH, Karl Erik. Normal variability of tonic vibration reflexes in man. Journal experimental neurology, v. 1, n. 16, p. 80-92, 1966.

HALLAL, Camilla Zamfolini; MARQUES, Nise Ribeiro; GONÇALVES, Mauro. O uso da vibração como método auxiliar no treinamento de capacidades físicas: uma revisão da literatura. Motriz, v. 16, n. 2 , p. 527-533, 2010.

MAEDA, Noriaki e colaboradores. Effect of whole-body-vibration training on trunk-muscle strength and physical performance in healthy adults: preliminary results of a randomized controlled trial. Journal physical medicine and rehabilitation, v. 25, n. 4, p. 357-363, 2016.

NG, Joseph K-F e colaboradores. EMG activity normalization for trunk muscles in subjects with and without back pain. Journal medicine and science in sports and exercise, v. 34, n. 7, p. 1082-1086, 2002.

OSAWA, Yasuke; OGUMA Yuko; ONISHI, Shohei. Effects of whole-body vibration training on bone-free lean body mass and muscle strength in young adults. Journal of sports science and medicine, v. 10, n. 1, p. 97-104, 2011. 
PALMER, Thomas; UHL, Timothy. Interday reliability of peak muscular power outputs on an isotonic dynamometer and assessment of active trunk control using the chop and lift tests. Journal of the athletic training, v. 46, n. 2, p. 150-159, 2011.

PERCHTHALER, Dennis e colaboradores. Acute effects of whole-body vibration on trunk and neck muscle activity in consideration of different vibration loads. Journal of sports science and medicine, v. 14, n. 1, p. 155-162, 2015.

REINHER, Fernanda Beatriz; CARPES, Felipe Pivetta; MOTA, Carlos Bolli. Influence of core stabilization training on low back pain and stability. Fisioterapia em movimento, v. 21, n. 1, p. 123-129, 2008.

RODRIGUES, Renata dos Santos, MENEZES, Nathanny Corrêa, WEIS; Luciana Cezimbra. Os benefícios da plataforma vibratória em pacientes portadores da doença de Parkinson: revisão bibliográfica. Salão de Ensino e de Extensão, 7. Anais.... Santa Cruz do Sul, BA: Unisc, 2016

ROELANTS, Machteld e colaboradores. Whole-body-vibration-induced increase in leg muscle activity during different squat exercises. Journal of strength conditioning research, v. $20, n$. 1, p. 124-129, 2006.

SHINOHARA, Minoru. Effects of prolonged vibration on motor unit activity and motor performance. Journal medicine and science in sports e exercise, v. 37, n. 12, p. 2120-2125, 2005.

TEIXEIRA, Cauê La Scala e colaboradores. Ten important facts about core training. ACSM's health \& fitness journal, v. 23, n. 1, p. 16-21, 2019.

TEYHEN, Deydre e colaboradores. Changes in deep abdominal muscle thickness during common trunk-strengthening exercises using ultrasound imaging. The journal of orthopaedic and sports physical therapy, v. 38, n. 10, p. 596-605, 2008.

VERSCHUEREN, Sabine e colaboradores. Effect of 6-month whole body vibration training on hip density, muscle strength and postural control in postmenopausal women: a randomized controlled pilot study. Journal of bone and mineral research, v. 19, n. 3, p. 352-359, 2004.

WIRTH, Brigitte; ZURFLUH, Stephan; MÜLLER, Roland. Acute effects of whole-body vibration on trunk muscles in young healthy adults. Journal of electromyography and kinesiology, $\mathrm{v}$. 28, n. 10, p. 450-457, 2011.

YOUDAS, James e colaboradores. Electromyographic analysis of gluteus maximus and hamstring activity during the supine resisted hip extension exercise versus supine unilateral bridge to neutral. Journal physiotherapy theory and practice, v. 33, n. 2, p. 124-130, 2017.

\section{Dados do primeiro autor:}

Email: victorcosta.vtf@gmail.com

Endereço: Av. Fernando Correa da Costa, 2367, Bairro Boa Esperança, Cuiabá, MT, CEP: 78060900, Brasil. 
Recebido em: 28/05/2021

Aprovado em: 15/07/2021

\section{Como citar este artigo:}

COUTINHO, Vitor da Costa e colaboradores. Atividade eletromiográfica de estabilizadores de tronco no exercício push-up isométrico com vibrações mecânicas. Corpoconsciência, v. 25, n. 2, p. 149-161, mai./ ago., 2021.

\section{Agradecimentos:}

Os autores agradecem à Fundação de Amparo à Pesquisa do Estado de Mato Grosso (FAPEMAT) por proporcionar a aquisição de recursos necessários para a realização desse estudo. 